\title{
MIXED MEANS AND HARDY'S INEQUALITY
}

\author{
A. ČǏ̌MEŠIJA AND J. PEČARIĆ
}

\begin{abstract}
Integral means of arbitrary order, with power weights, and their companion means are introduced and related mixed-means inequalities are derived. These results are then used in proving inequalities of Hardy and Levin-Cochran-Lee type. Also, new proofs of Hardy and Carleman inequality for finite and infinite series are given by using discrete mixed-means.
\end{abstract}

Mathematics subject classification (1991): 26D10, 26D15. inequality.

Key words and phrases: Mixed-means, Hardy inequality, Levin-Cochran-Lee inequality, Carleman

\section{REFERENCES}

[1] M. Alić and J. Pečarić, Inequalities related to Hardy's and Levin's, Rad Hrvatske akad. znan. umj. mat. [467] 11 (1994), 111-117.

[2] N. G. De Bruijn, Carleman's inequality for finite series, Nederl. Akad. Wet. Amsterdam. Proc. Ser. A. 44 (1963), 505-514 (= Indagationes Mathematicae 34).

[3] P. S. Bullen, A Chapter on Inequalities, Math. Medley 21, No. 2 (1993), 48-69.

[4] P. S. Bullen, Inequalities Due to T. S. Nanjundiah, manuscript

[5] J. A. Cochran and C.-S. Lee, Inequalities related to Hardy's and Heinig's, Math. Proc. Cambridge Phil. Soc. 96 (1984), 1-7.

[6] G. Hardy, J. E. Littlewood and G. Pólya, Inequalities, second edition, Cambridge University Press, Cambridge, 1967.

[7] F. Holland, On a mixed arithmetic-mean, geometric-mean inequality, Mathematics Competitions 5 (1992), 60-64.

[8] K. Kedlaya, Proof of a Mixed Arithmetic-Mean, Geometric-Mean Inequality, Amer. Math. Monthly, 101 (1994), 355-357.

[9] V. Levin, O neravenstvah III: Neravenstva, vypolnjaemie geometričeskim srednim neotricatel'noi funkcii, Math. Sbornik 4 (46) (1938), 325-331.

[10] E.R. Love, Inequalities related to those of Hardy and of Cochran and Lee, Math. Proc. Cambridge Phil. Soc. 99 (1986), 395-408.

[11] A. Lupaş, Asupra problemei 579 (1901) din Gazeta Matematica, Gaz. Mat. (Bucharest) 81 (1976), 281-286.

[12] T. Matsuda, An Inductive Proof of a Mixed Arithmetic-Geometric Mean Inequality, Amer. Math. Monthly 102 (1995), 634-637.

[13] D. S. Mitrinović, J. E. Pečarić and A. M. Fink, Inequalities Involving Functions and Their Integrals and Derivatives, Kluwer Academic Publishers, 1991.

[14] B. Mond and J. Pečarić, A Mixed means Inequality, Austral. Math. Soc. Gazette, 23 (1996), No. 2, $67-70$.

[15] B. Mond and J. Pečarić, A Mixed Arithmetic-Mean-Harmonic-Mean Matrix Inequality, Linear Algebra Appl. 237/238 (1996), 449-454.

[16] B. Mond and J. Pečarić, Mixed means inequalities for positive linear operators, Austral. Math. Soc. Gazette, 23 (1996), No. 5, 198-200.

[17] T. S. Nanjundiah, Sharpening some classical inequalities, Math. Student, 20 (1952), 24-25. 
[18] W. Rudin, Real and Complex Analysis, McGraw-Hill, 1970.

[19] Gou-Sheng Yang, Yu-Jen Lin, On companion inequalities related to Heinig's, Tamkang Journal of Math., 22 (1991), No. 4, 313-322.

[20] H. S. Wilf, On finite sections of the classical inequalities. Nederl. Akad. Wet. Amsterdam. Proc. Ser. A. 65 (1962), 340-342 (Indagationes Mathematicae 24). 